Disclosure of Interests: None declared

DOI: 10.1136/annrheumdis-2019-eular.7910

\section{AB0660 $\quad$ SEXUAL HEALTH IMPAIRMENT IN WOMEN WITH IDIOPATHIC INFLAMMATORY MYOPATHIES}

Barbora Heřmánková ${ }^{1}$, Maja Špiritović ${ }^{1}$, Hana Smucrova ${ }^{2}$, Sabina Oreska $^{2,3}$ Hana Štorkánová ${ }^{2,3}$, Kristyna Bubova ${ }^{2,3}$, Karel Pavelka ${ }^{2,3}$, Jiř́ Vencovsky $\hat{y}^{2,3}$, Ladislav Šenolt ${ }^{2,3}$, Heřman Mann ${ }^{2,3}$, Michal Tomčík ${ }^{2,3}$. ${ }^{1}$ Faculty of Physical Education and Sport, Charles University, Prague, Czech Republic; ${ }^{2}$ Institute of Rheumatology, Prague, Czech Republic; ${ }^{3} 1$ st Faculty of Medicine, Charles University, Department of Rheumatology, Prague, Czech Republic

Background: Idiopathic inflammatory myopathies (IIM) are a group of disorders characterized by skeletal muscle inflammation that can cause functional impairment including sexual dysfunction.

Objectives: To assess sexual function, pelvic floor function and sexual quality of life of women with IIM compared to age-/sex-matched healthy controls $(\mathrm{HC})$. To subanalyze sexual function in sexually active individuals.

Methods: In total 27 women with IIM [mean age: 54.2, disease duration: 7.3 years, dermatomyositis (DM, 10)/polymyositis (PM, 13)/necrotizing myopathy (IMNM, 3)/inclusion body myositis (IBM, 1)], who fulfilled the Bohan/Peter 1975 criteria for DM/PM, and 27 healthy women (mean age: 54.2) filled in 11 well-established and validated questionnaires assessing sexual function, pelvic floor function, quality of life, fatigue, physical activity and depression. Data are presented as mean \pm SEM.

Results: Compared to HC, patients with IIM had significantly higher prevalence and greater severity of sexual impairment (FSFI, BISF-W), dysfunction of pelvic floor (PISQ-12), and worse sexual quality of life (SQoLF) (table 1). There were no significant differences in sexual function between PM and DM. Even sexually active IIM patients reported significantly greater sexual health impairment compared to sexually active HC. Sexual health impairment in IIM was associated with laboratory markers of disease activity, health status, physical activity, fatigue and depression (table 2).

Table 1.

\begin{tabular}{|c|c|c|c|c|c|c|}
\hline $\begin{array}{l}\text { Questionnaire: } \\
\text { score range }\end{array}$ & $\underset{(n=27)}{\operatorname{IIM}}$ & $\begin{array}{c}\mathrm{HC} \\
(\mathrm{n}=27)\end{array}$ & p-value & $\begin{array}{l}\text { IIM SA } \\
(n=16)\end{array}$ & $\begin{array}{l}\text { HC SA } \\
(n=16)\end{array}$ & p-value \\
\hline $\begin{array}{l}\text { FSFI :2(worst)-36 } \\
\text { (best) }\end{array}$ & $\begin{array}{l}15.7 \\
\pm 2.5\end{array}$ & $\begin{array}{l}23.0 \\
\pm 2.2\end{array}$ & $\mathrm{p}=0.0296$ & $23.8 \pm 2.6$ & $29.6 \pm 0.8$ & ns \\
\hline $\begin{array}{l}\text { BISF-W:-16(worst)- } \\
75 \text { (best) }\end{array}$ & $\begin{array}{r}15.9 \\
\pm 3.5\end{array}$ & $\begin{array}{l}27.4 \\
\pm 3.5\end{array}$ & $\mathrm{p}=0.0212$ & $25.2 \pm 4.3$ & $39.2 \pm 2.3$ & $\mathrm{p}=0.0151$ \\
\hline $\begin{array}{l}\text { SQoL-F: } 0 \text { (worst)- } \\
100 \text { (best) }\end{array}$ & $\begin{array}{l}21.5 \\
\pm 4.5\end{array}$ & $\begin{array}{l}83.3 \\
\pm 2.9\end{array}$ & $p<0.0001$ & $31.9 \pm 6.4$ & $85.7 \pm 3.0$ & $\mathrm{p}<0.0001$ \\
\hline $\begin{array}{l}\text { PISQ-12: 0(best)-48 } \\
\text { (worst) }\end{array}$ & $\begin{array}{l}14.0 \\
\pm 1.0\end{array}$ & $8.7 \pm 0.9$ & $p=0.0007$ & $11.4 \pm 1.1$ & $9.2 \pm 1.0$ & ns \\
\hline $\begin{array}{l}\text { PFIQ7: 0(best)-300 } \\
\text { (worst) }\end{array}$ & $\begin{array}{l}20.5 \\
\pm 6.9\end{array}$ & $6.0 \pm 2.1$ & ns & $17.6 \pm 10.8$ & $10.4 \pm 4.2$ & ns \\
\hline
\end{tabular}

Abbreviations: FSFI: Female Sexual Function Index; BISF-W: Brief Index of Sexual Function for Women; SQoL-F: Sexual Quality of Life Questionnaire; PISQ-12: Pelvic Organ Prolapse/ Urinary Incontinence Sexual Questionnaire short form; PFIQ7: Pelvic Floor Distress Inventory Questionnaire, SA: sexually active; ns: not significant

Table 2.

\begin{tabular}{lccc}
\hline Parameter: score range & Correlated parameters & r & p-value \\
\hline FIS (fatigue): 0(best)-160(worst) & FSFI (sexual function) & -0.406 & $\mathrm{p}=0.0353$ \\
& BISF-W (sexual function) & -0.424 & $\mathrm{p}=0.0272$ \\
& SQol-F (sexual quality of life) & -0.562 & $\mathrm{p}=0.0027$ \\
& PISQ-12 (pelvic floor & 0.459 & $\mathrm{p}=0.0157$ \\
& function) & & \\
HAP (physical activity): 0(worst)-94 & FSFI (sexual function) & 0.429 & $\mathrm{p}=0.0252$ \\
(best) & FSFI (sexual function) & -0.411 & $\mathrm{p}=0.0327$ \\
LD & BISF-W (sexual function) & -0.466 & $\mathrm{p}=0.0141$ \\
BDI II (depression): 0(best)-63(worst) & PISQ-12 (pelvic floor & 0.322 & $\mathrm{p}=0.0400$ \\
& $\quad$ function) & & \\
HAQ (health status): 0(best)-3(worst) & PISQ-12 (pelvic floor & 0.477 & $\mathrm{p}=0.0117$ \\
& $\quad$ function) & & \\
& BISF-W (sexual function) & -0.411 & $\mathrm{p}=0.0329$
\end{tabular}
Abbreviations: FIS: Fatigue Impact Scale; BDI-II: Beck's Depression Inventory II; HAP: Human Activity Profile; HAQ: Health Assessment Questionnaire; LD: lactate dehydrogenase Conclusion: Women with IIM reported significantly impaired sexual function, sexual quality of life and pelvic floor function than age-matched HC.
Worse scores in IIM were associated with disease activity/health status, physical activity, fatigue and depression.Acknowledgement: Supported by AZV-16-33574A, MHCR 023728.

Disclosure of Interests: Barbora Heřmánková: None declared, Maja Špiritović: None declared, Hana Smucrova: None declared, Sabina Oreska: None declared, Hana Štorkánová: None declared, Kristyna Bubova: None declared, Karel Pavelka: None declared, Jiří Vencovský Consultant for: Samsung, Speakers bureau: AbbVie, Novartis, Pfizer, Sanofi, Eli Lilly, Biogen, UCB, MSD, Werfen, Roche, Ladislav Šenolt Grant/research support from: AbbVie, Consultant for: AbbVie, Bristol-Myers Squibb, Celgene Corporation, Merck Sharp and Dohme, Novartis, Pfizer, Roche, UCB, Amgen, Takeda, Speakers bureau: AbbVie, Amgen, Bristol-Myers Squibb, Celgene Corporation, Eli Lilly, Merck Sharp and Dohme, Novartis, Pfizer, Roche UCB, Heřman Mann Consultant for: Pfizer, Eli Lilly, Sanofi, Speakers bureau: AbbVie, Roche, Pfizer, MSD, Eli Lilly, Sanofi, Michal Tomčík: None declared

DOI: 10.1136/annrheumdis-2019-eular.7358

\section{AB0661 ASSOCIATION OF ANTI-MDA-5 AUTOANTIBODY WITH AUTOIMMUNE ASSOCIATED HEMOPHAGOCYTIC SYNDROMEIN DERMATOMYOSITIS}

Manabu Honda ${ }^{1}$, Mayuko Moriyama ${ }^{2}$, Masahiro Kondo ${ }^{2}$, Shunichi Kumakura ${ }^{1}$, Yoshiko Sumita ${ }^{2}$, Yohko Murakawa ${ }^{2}$. ${ }^{1}$ Shimane University Faculty of Medicine, Department of Medical Education and Research, Izumo, Japan; ${ }^{2}$ Shimane University Faculty of Medicine, Department of Rheumatology, Izumo, Japan

Background: Autoimmune associated hemophagocytic syndrome (AAHS) is a rare complication in dermatomyositis (DM). We previously demonstrated by multivariate analysis that one of factors associated with mortality in AAHS is DM (OR 5.57 [95\% Cl 1.08-28.65], P 0.05) among connective tissue diseases (1).

Objectives: To find out underlying immunological characteristics, we examined the DM patients with AAHS

Methods: We examined 31 new onset patients with idiopathic inflammatory myopathies (IIM) including clinically amyopathic dermatomyositis (CADM) admitted to our hospital between January 2009 and December 2018. Three patients had been diagnosed as AAHS proven by bone marrow aspiration. We examined these patient clinical and laboratory characteristics.

Results: Two patients were diagnosed as DM and one was CADM. We found that these 3 patients were associated with interstitial pneumonia Laboratory tests of all 3 patients showed hyperferritinemia and high titer of anti-MDA5 antibody. All patients were diagnosed as AAHS by bone marrow aspiration smears. Two of them died on the 12th and on the 75th hospital day, respectively, in spite of intensive therapies.

\begin{tabular}{|c|c|c|c|c|c|c|c|c|}
\hline & \multirow{2}{*}{$\begin{array}{l}\text { age/ } \\
\text { sex }\end{array}$} & \multirow[t]{2}{*}{ diag. } & \multirow{2}{*}{$\mathrm{CK}(\mathrm{n})$} & \multirow{2}{*}{ ferritin (n) } & \multicolumn{4}{|c|}{ antibodies } \\
\hline & & & & & $\overline{\mathrm{MDA5}}$ & Ro & $\begin{array}{c}\text { PM- } \\
\text { SCL100 }\end{array}$ & others* \\
\hline 1 & $63 / F$ & DM & $\begin{array}{c}8430(36- \\
216)\end{array}$ & $\begin{array}{c}59,877(5- \\
120)\end{array}$ & $>150$ & + & - & - \\
\hline 2 & $50 / \mathrm{M}$ & CIDM & $32(59-248)$ & $\begin{array}{c}11,498(23- \\
250)\end{array}$ & $>150$ & \pm & \pm & - \\
\hline 3 & $57 / F$ & DM & $277(41-153)$ & $4,876(5-120)$ & $>150$ & + & - & - \\
\hline
\end{tabular}

Conclusion: Anti-MDA5 may relate not only interstitial pneumonia but also AAHS.

\section{REFERENCES}

[1] 1. Kumakura S and Murakawa Y: Arthritis Rheum 2014;66:2297

Disclosure of Interests: Manabu Honda Speakers bureau: Asahi Kase Pharma, Ono Pharm., Mayuko Moriyama: None declared, Masahiro Kondo Speakers bureau: Eisai Co., Chugai Pharma, Mitsubishi Tanabe Pharma, Bristol-Myers Squibb, Janssen Pharma, Astellas Pharma, Takeda Pharma NIPPON KAYAKU, Daiichi-Sankyo, Asahi Kasei Pharma, AbbVie Japan. Shunichi Kumakura: None declared, Yoshiko Sumita: None declared, Yohko Murakawa Grant/research support from: Asahi Kasei Pharmaceutical Co., Ltd.

Chugai Pharmaceutical Co., Ltd.

Ono Pharmaceutical Co., Ltd.

Daiichi Sankyo Co., Ltd.

Teijin Pharma Ltd. 